\title{
AN EXAMPLE PERTAINING TO THE FAILURE OF THE BESICOVITCH-FEDERER STRUCTURE THEOREM IN HILBERT SPACE
}

\author{
Thierry De Pauw
}

\begin{abstract}
We give an example, in the infinite dimensional separable Hilbert space, of a purely unrectifiable Borel set with finite nonzero one dimensional Hausdorff measure, whose projection is nonnegligible in a set of directions which is not Aronszajn null.
\end{abstract}

2010 Mathematics Subject Classification: 28A75, 28A80, 53C65.

Key words: Rectifiable set, purely unrectifiable set, Hilbert space, Negligible set.

\section{Foreword}

We let $0<m<n$ be integers. The Grassmannian $\mathbf{G}\left(\mathbb{R}^{n}, m\right)$ is equipped with an $\mathbf{O}(n)$ invariant probability Borel measure $\gamma_{n, m}[\mathbf{1 1}$, 2.7.16(6)]. Given $W \in \mathbf{G}\left(\mathbb{R}^{n}, m\right)$ we let $P_{W}$ denote the orthogonal projection onto $W$. The Besicovitch-Federer Theorem referred to in the title states the following: If $S \subseteq \mathbb{R}^{n}$ is Borel measurable and $\mathscr{H}^{m}(S)<\infty$ then the following are equivalent:

(A) $\mathscr{H}^{m}(S \cap M)=0$ for each $m$ dimensional $C^{1}$ submanifold $M \subseteq \mathbb{R}^{n}$. (B) $\mathscr{H}^{m}\left(P_{W}(S)\right)=0$ for $\gamma_{n, m}$ almost every $W \in \mathbf{G}\left(\mathbb{R}^{n}, m\right)$.

Here $\mathscr{H}^{m}$ is the $m$ dimensional Hausdorff measure in $\mathbb{R}^{n}$. The $m$ rectifiable subsets of $\mathbb{R}^{n}$ are defined to be those of the form $M=f(A)$ for some bounded $A \subseteq \mathbb{R}^{m}$ and Lipschitz $f: A \rightarrow \mathbb{R}^{n}$. It follows from Theorems of H. Rademacher [11, 3.1.6], N. Luzin [11, 2.3.5], and H. Whitney $[\mathbf{1 1}, 3.1 .14]$, that condition (A) is equivalent to $\left(\mathrm{A}^{\prime}\right) \mathscr{H}^{m}(S \cap M)=0$ for every $m$ rectifiable subset $M \subseteq \mathbb{R}^{n}$.

The Structure Theorem was originally proved by A. S. Besicovitch [3] in case $n=2$ and $m=1$, and generalized to arbitrary $n$ and $m$ by H. Federer $[\mathbf{1 0}]$. E. J. Mickle [19] gave an improved version on which $[\mathbf{1 1}$, 
3.3.14] and [18, 18.10(2)] report. Quantitative versions of the statement have been discussed for instance by T. C. O'Neil [20], G. David and S. Semmes $[6]$, and T. Tao $[\mathbf{2 3}]$. B. White $[\mathbf{2 5}]$ has shown that the general result follows by elementary means from the Besicovitch $n=$ $m+1=2$ case.

The Besicovitch-Federer Theorem has played a distinguished role in the development of the Geometric Calculus of Variations, of which the Plateau problem is a paradigm. The original proof of the Closure Theorem for integral currents due to H. Federer and W. H. Fleming [13] relies upon the Structure Theorem, and so does one more recent proof, perhaps (unfortunately) less known, due to W. H. Fleming [14], see also [12]. Other proofs of the Closure Theorem have avoided the Structure Theorem, see the techniques set forth in [1] and [21], as well as [24], whether these have been designed to this end or not. Even more recent versions of the Closure Theorem, when the ambient space $\mathbb{R}^{n}$ is replaced with either a Banach space or a complete metric space, rely upon the fact that 0 dimensional slices of integral currents are of bounded variation - an observation that goes back at least to H. Federer's [11, 5.3.5(1)].

However it has been so far unknown whether a version of the Structure Theorem holds when the ambient space $\mathbb{R}^{n}$ is replaced with a separable infinite dimensional Banach space, for instance the simplest one, $\ell_{2}$. Complications in stating the problem soon arise: Even though orthogonal projections $P_{W}$ onto $W \in \mathbf{G}\left(\ell_{2}, m\right)$ make sense in the Hilbert setting, one needs to face the nonexistence of an invariant probability measure on the infinite dimensional Grassmannian $\mathbf{G}\left(\ell_{2}, m\right)$. Nevertheless stating condition (B) above does not require the existence of such measure, but merely the existence of a distinguished invariant $\sigma$ ideal of null sets in $\mathbf{G}\left(\ell_{2}, m\right)$ (see also the forthcoming [4]). This puts us in a better position as we explain hereunder.

From now on we shall consider the case $m=1$ only. The projective space $\mathbf{G}\left(\ell_{2}, 1\right)$ is the usual quotient of the unit sphere $S_{\ell_{2}}$. In fact the map

$$
\psi: \ell_{2} \backslash\{0\} \rightarrow \mathbf{G}\left(\ell_{2}, 1\right): v \mapsto \operatorname{span}\{v\}
$$

allows us to push-forward any $\sigma$ ideal from $\ell_{2}$ to $\mathbf{G}\left(\ell_{2}, 1\right)$, as one would do with a measure. Replacing temporarily $\ell_{2}$ by $\mathbb{R}^{n}$ in this construction, and recalling that the $\mathbf{O}(n)$ invariant measure $\gamma_{n, 1}$ is a normalized quotient of $\mathscr{H}^{n-1}\left\llcorner\mathbb{S}^{n-1}\right.$, we infer from integration in polar coordinates that $\gamma_{n, 1}(E)=0$ if and only if $\mathscr{L}^{n}\left(\psi^{-1}(E)\right)=0$. Accordingly, a sought for $\sigma$ ideal of null sets in $\mathbf{G}\left(\ell_{2}, 1\right)$ can be obtained by a $\psi$ push-forward of some $\sigma$ ideal of null sets in $\ell_{2}$ generalizing the Lebesgue null sets of $\mathbb{R}^{n}$. 
There are several such choices. In this paper we consider Aronszajn null sets in $\ell_{2}$ (see Subsection 1.4 for the definition), which are known to be equivalent to cube null and Gaussian null sets [5], but are not equivalent to Haar null sets, see e.g. the instructive monograph [2], which also discusses the relevance of Aronszajn null sets for instance to the almost everywhere Gâteaux differentiability of Lipschitz functions $\ell_{2} \rightarrow \mathbb{R}$.

Those $S \subseteq \mathbb{R}^{n}$ verifying condition $\left(\mathrm{A}^{\prime}\right)$ above are termed purely $\left(\mathscr{H}^{m}, m\right)$ unrectifiable. The definition makes sense in any ambient metric space, in particular in $\ell_{2}$. Possibly the simplest and most classical example of a purely $\left(\mathscr{H}^{1}, 1\right)$ unrectifiable subset of $\mathbb{R}^{2}$ is the self-similar four corners Cantor set $C$ illustrated in Figure 1. This paper contributes the following:

Theorem. There exists a purely $\left(\mathscr{H}^{1}, 1\right)$ unrectifiable Borel measurable subset $S \subseteq \ell_{2}$ with $\mathscr{H}^{1}(S)=1$, such that the set of directions from which $S$ is visible,

$$
\ell_{2} \cap\left\{v: v \neq 0 \text { and } \mathscr{H}^{1}\left(P_{\operatorname{span}\{v\}}(S)\right)>0\right\}
$$

is not Aronszajn null.

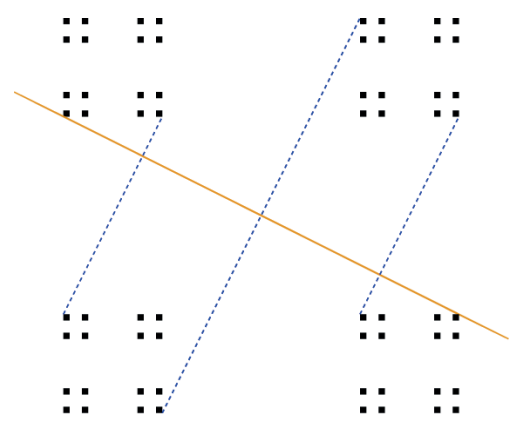

Figure 1. Four corners Cantor set in Euclidean plane.

We now briefly indicate why this is the case, and Section 2 consists of a detailed version of the argument. It is relevant to observe on Figure 1 that there actually exist "exceptional" lines on which $C$ projects to a nonnegligible set - it projects for instance to a nondegenerate interval $I$ on the orange line $L$. As a matter of fact the restriction of the projection $P_{L}\lceil C$ is nearly injective, so that $C$ is nearly a graph. Specifically, if we remove the corners of the countably many solid squares used in the inductive construction of $C$, we obtain a set $\tilde{C}$ which is the graph of $f: I \backslash D \rightarrow L^{\perp}$, where $D$ is countable and $f=P_{L^{\perp}} \circ\left(P_{L} \backslash \tilde{C}\right)^{-1}$. 
By general descriptive set theory $f$ is Borel measurable. In fact $f$ is continuous, as the happy reader will verify. However $f$ cannot possibly be Lipschitz, for otherwise $C$ would be 1 rectifiable - nor even approximately differentiable on a set of positive measure, for otherwise $C$ would not be purely $\left(\mathscr{H}^{1}, 1\right)$ unrectifiable.

Figure 1 suggests to consider a sequence $\left\langle f_{j}\right\rangle_{j}$ of step functions approximating $f$, in the obvious way when $f_{1}$ takes four distinct values, $f_{2}$ takes sixteen distinct values, etc. Details are provided in Subsection 2.1. We use these to define

$$
\gamma: I \rightarrow \ell_{\infty}: t \mapsto\left(t, f_{1}(t), f_{2}(t), \ldots\right)
$$

The fact that $f$ is approximately differentiable almost nowhere should somehow imply the same about $\gamma$. This in turn should say that $S=\operatorname{im} \gamma$ is purely $\left(\mathscr{H}^{1}, 1\right)$ unrectifiable. In fact the domain $I$ of $f_{j}$ is divided into $4^{j}$ intervals $I_{j, k}$ on which $f_{j}$ is constant, and if we define

$$
\delta_{j}(t)=\frac{\operatorname{dist}\left(t, \cup_{k} \operatorname{bdry} I_{j, k}\right)}{4^{-j}},
$$

$t \in I$, then the reader will easily apply the proof of Subsection 2.5 to showing that if $\inf _{j} \delta_{j}(t)=0$ then $\gamma$ is not approximately differentiable at $t$, as should be intuitively sound. Furthermore the Lebesgue density Theorem clearly implies that

$$
I \cap\left\{t: \inf _{j} \delta_{j}(t)=0\right\}
$$

is conegligible in $I$. Therefore $S=\operatorname{im} \gamma$ is purely $\left(\mathscr{H}^{1}, 1\right)$ unrectifiable, because $\gamma$ has the Luzin (N) property (adapt Subsections 2.3 and $2.6^{1}$ ). Yet the projection of $S=\operatorname{im} \gamma$ onto a subspace generated by finitely many coordinates, consists of finitely many line segments, a "very" rectifiable set whose projections should have positive measure in many directions, if only we had made sense of such statement in $\ell_{\infty}$.

We now seek for a modification of $\gamma$ taking their values in $\ell_{2}$ rather than in $\ell_{\infty}$. With each sequence $\left\langle\beta_{j}\right\rangle_{j} \in \ell_{2}$ we can of course associate

$$
\gamma: I \rightarrow \ell_{2}: t \mapsto\left(t, \beta_{1} f_{1}(t), \beta_{2} f_{2}(t), \ldots\right)
$$

The problem is that by doing so we have likely destroyed the almost everywhere non approximate differentiability property that we used to imply the image of $\gamma$ is purely unrectifiable. This is because $\beta_{j} \rightarrow 0$. Hope suggests to investigate the case when this convergence is slow. The point here is that the coordinates $\left\langle f_{j}\right\rangle_{j}$ are stochastically independent,

\footnotetext{
${ }^{1}$ Here one cannot rely upon Subsection 1.2 as $\ell_{\infty}$ does not have the Radon-Nikodým property, and one needs to argue solely with weak limits of difference quotients.
} 
thus the Borel-Cantelli Lemma yields an improved version of the use of the Lebesgue density Theorem above, see Subsection 2.7. In Subsection 2.8 we establish the existence of a proper choice of $\left\langle\beta_{j}\right\rangle_{j} \in \ell_{2}$ such that our new $\gamma$ is approximately differentiable almost nowhere.

It may be worth pointing out that this can be interpreted as a stronger version of the nondifferentiability property of the original function $f$ arising in $\mathbb{R}^{2}$. In other words, the four corners Cantor set of Figure 1 is very much purely unrectifiable, in some yet unspecified sense. Whether or not this is generic behavior of purely unrectifiable subsets of the Euclidean plane remains unsettled.

We finally need to evoke why $S=\operatorname{im} \gamma$ projects to a set of positive measure onto "many" lines $L=\operatorname{span}\{u\}, u \in S_{\ell_{2}}$. From the definition of $\gamma$ we infer that the measure of this projection equals the measure of the image of

$$
h_{u}(t)=\langle\gamma(t), u\rangle=u_{0} t+\sum_{j=1}^{\infty} \beta_{j} u_{j} f_{j}(t) .
$$

Even though we arranged everything so far in order that $t \mapsto \sum_{j \geqslant 1} \beta_{j} f_{j}(t)$ be very much nonLipschitz, we can now play with the coefficients $\left\langle u_{j}\right\rangle_{j}$, hopefully allowed to converge fast enough to 0 , to compensate for this wild behavior, in fact to guarantee $t \mapsto \sum_{j \geqslant 1} \beta_{j} u_{j} f_{j}(t)$ is Lipschitz with small Lipschitz constant, when restricted to an appropriate nonnegligible subset of its domain. This associated with the choice of a first coordinate $u_{0} t$ with $u_{0}$ close to 1 yields a projection of positive measure, for Aronszajn nonnegligeably many $u$ 's, see Subsection 2.10.

\section{Preliminaries}

The ambient space of this paper is the infinite dimensional separable Hilbert space $\ell_{2}(\mathbb{N})$, sometimes abbreviated $\ell_{2}$. We let $e_{0}, e_{1}, e_{2}, \ldots$ be its canonical orthonormal basis. The norm in $\ell_{2}$ is denoted $\|\cdot\|$ or $\|\cdot\|_{\ell_{2}}$, and the inner product $\langle\cdot, \cdot\rangle$. Occasionally we consider the finite dimensional $\ell_{2}^{n}$, i.e. $\mathbb{R}^{n}$ equipped with its usual inner product. Whether $X=\ell_{2}$ or $X=\ell_{2}^{n}$ we let $S_{X}$ denote the unit sphere of $X$.

1.1. Hausdorff measure. Given $S \subseteq \ell_{2}(\mathbb{N})$ and $\delta>0$ we recall that

$$
\begin{array}{r}
\mathscr{H}_{\delta}^{1}(S)=\inf \left\{\sum_{j \in J} \operatorname{diam} S_{j}: S \subseteq \bigcup_{j \in J} S_{j}, J\right. \text { is at most countable, } \\
\text { and } \left.\operatorname{diam} S_{j} \leqslant \delta \text { for every } j\right\},
\end{array}
$$


and

$$
\mathscr{H}^{1}(S)=\sup _{\delta>0} \mathscr{H}_{\delta}^{1}(S) .
$$

1.2. Differentiability of Lipschitz maps. If $a<b$ and $f:[a, b] \rightarrow$ $\ell_{2}(\mathbb{N})$ is Lipschitz, then $f$ is differentiable $\mathscr{L}^{1}$ almost everywhere. This is well-known and will be used in Subsection 2.6. As it also happens to be easily established, we include a sketch of proof.

For each $x \in \ell_{2}(\mathbb{N})$ we define $f_{x}:[a, b] \rightarrow \mathbb{R}$ by $f_{x}(t)=\langle f(t), x\rangle$ and we notice $\operatorname{Lip} f_{x} \leqslant \operatorname{Lip} f$. Note also that

$$
\left|f_{x}^{\prime}(t)-f_{y}^{\prime}(t)\right| \leqslant(\operatorname{Lip} f)\|x-y\|
$$

whenever both $f_{x}$ and $f_{y}$ are differentiable at $t$. Choose a dense sequence $\left\langle x_{j}\right\rangle_{j}$ in $\ell_{2}(\mathbb{N})$ and let $N_{j}$ be an $\mathscr{L}^{1}$ negligible subset of $(a, b)$ such that $f_{x_{j}}$ is differentiable at each $t \in(a, b) \backslash N_{j}$. Put $N=\cup_{j} N_{j}$. It easily follows from (1) that for each $t \in(a, b) \backslash N$ the difference quotients

$$
\frac{f(t+h)-f(t)}{h}
$$

converge weakly in $\ell_{2}(\mathbb{N})$, and we denote the corresponding limit by $g(t)$. We ought to show that the convergence to $g(t)$ is in fact strong for $\mathscr{L}^{1}$ almost every $t$. Recall that the weak convergence is promoted to strong convergence when norms converge as well, according to the parallelogram law. Since readily

$$
\|g(t)\| \leqslant \liminf _{h \rightarrow 0}\left\|\frac{f(t+h)-f(t)}{h}\right\|
$$

we merely need to identify those $t \in(a, b) \backslash N$ such that

$$
\limsup _{h \rightarrow 0}\left\|\frac{f(t+h)-f(t)}{h}\right\| \leqslant\|g(t)\| .
$$

Note the function $t \mapsto\|g(t)\|$ is $\mathscr{L}^{1}$ measurable. Observe next that for every $t, t+h \in[a, b]$ and every $j$ one has

$$
\begin{aligned}
\left\langle f(t+h)-f(t), x_{j}\right\rangle=\int_{t}^{t+h} f_{x_{j}}^{\prime} d \mathscr{L}^{1}= & \int_{t}^{t+h}\left\langle g(s), x_{j}\right\rangle d \mathscr{L}^{1}(s) \\
& \leqslant\left\|x_{j}\right\| \int_{t}^{t+h}\|g(s)\| d \mathscr{L}^{1}(s) .
\end{aligned}
$$

Extracting from $\left\langle x_{j}\right\rangle_{j}$ a sequence that converges to $f(t+h)-f(t)$ we infer from the above that

$$
\|f(t+h)-f(t)\| \leqslant \int_{t}^{t+h}\|g\| d \mathscr{L}^{1} .
$$

It is now clear that (2) holds whenever $t$ is a Lebesgue point of $\|g\|$. 
1.3. Pure unrectifiability. We say $R \subseteq \ell_{2}(\mathbb{N})$ is 1 rectifiable if there exists a bounded set $A \subseteq \mathbb{R}$ and a Lipschitz map $f: A \rightarrow \ell_{2}(\mathbb{N})$ such that $R=f(A)$. We say $S \subseteq \ell_{2}(\mathbb{N})$ is purely $\left(\mathscr{H}^{1}, 1\right)$ unrectifiable if $\mathscr{H}^{1}(S \cap R)=0$ for every 1 rectifiable set $R \subseteq \ell_{2}(\mathbb{N})$.

Using a Whitney decomposition of $\mathbb{R} \backslash \operatorname{clos} A$ one shows that each $f$ as above admits a Lipschitz extension $\hat{f}: \mathbb{R} \rightarrow \ell_{2}(\mathbb{N})$, see e.g. [15]. This follows alternatively from Kirszbraun's Theorem $[\mathbf{1 1}, 2.10 .43]$ or [2, Chapter 1, §2]. Furthermore the image of each restriction $\hat{f}\lceil[a, b]$ being pathwise connected is also arcwise connected, see e.g. [8, Problem 6.3.11], i.e. there exists an injective continuous $h:[a, b] \rightarrow \ell_{2}(\mathbb{N})$ such that $h(a)=\hat{f}(a), h(b)=\hat{f}(b)$, and $h[a, b] \subseteq \hat{f}[a, b]$. It follows that $\varphi:[a, b] \rightarrow\left[0, \mathscr{H}^{1}(h[a, b])\right]: t \mapsto \mathscr{H}^{1}(h[a, t])$ is a homeomorphism. Thus $\tilde{h}:=h \circ \varphi^{-1}$ is an arc in $\hat{f}[a, b]$ with $\left\|\tilde{h}\left(s_{1}\right)-\tilde{h}\left(s_{2}\right)\right\| \leqslant \mathscr{H}^{1}\left(\tilde{h}\left[s_{1}, s_{2}\right]\right)=$ $\left|s_{1}-s_{2}\right|$ whenever $0 \leqslant s_{1}<s_{2} \leqslant \mathscr{H}^{1}(h[a, b])$. Therefore Lip $\tilde{h} \leqslant 1$ and in turn $\left\|\tilde{h}^{\prime}(s)\right\| \leqslant 1$ for $\mathscr{L}^{1}$ almost every $s$. The Area Theorem from [17] then implies that $\left\|\tilde{h}^{\prime}(s)\right\|=1$ for $\mathscr{L}^{1}$ almost every $s$. This will be called an arclength parametrization.

1.4. Aronszajn null sets. Let $X$ be a separable Banach space. A Borel subset $B \subseteq X$ is Aronszajn null if the following holds. For every sequence $\left\langle v_{j}\right\rangle_{j}$ whose span is dense in $X$, there exists a decomposition $B=\cup_{j} B_{j}$ into Borel sets $B_{j}$ subject to the following requirement: For each $j$, the intersection of $B_{j}$ with each line parallel to $v_{j}$ is negligible, i.e. $\mathscr{H}^{1}\left(B_{j} \cap\left(y+\operatorname{span}\left\{v_{j}\right\}\right)\right)=0$ for every $y \in X$. We say that an arbitrary subset of $X$ is Aronszajn null if it is contained in an Aronszajn null Borel subset of $X$.

It is important in this definition that the sets $B_{j}$ be Borel measurable. Indeed W. Sierpiński established, under the continuum hypothesis, the existence of a partition $\mathbb{R}^{2}=E_{1} \cup E_{2}$ such that $E_{1}$ (respectively $E_{2}$ ) is null on every horizontal (respectively vertical) line, see e.g. [16, Chapter 4]. It follows from Fubini's Theorem applied to the Lebesgue measure $\mathscr{L}^{2}$ that one of $E_{1}$ and $E_{2}$ - and therefore both - must be $\mathscr{L}^{2}$ nonmeasurable.

It also follows from Fubini's Theorem applied to Lebesgue's measure $\mathscr{L}^{n}$, and from the Borel regularity of $\mathscr{L}^{n}$, that in case $X=\mathbb{R}^{n}$ is finite dimensional the Aronszajn null sets coincide with the Lebesgue null sets.

We now let $X$ be a separable Hilbert space, i.e. $X=\ell_{2}^{n}$ for some $n \in \mathbb{N}$ or $X=\ell_{2}$. By $\mathbf{G}(X, 1)$ we denote the collection of 1 dimensional linear subspaces of $X$. We consider the hereditary $\sigma$ ideal $\mathscr{N}_{X}$ consisting 
of those Aronszajn null sets in $X$. We use the map

$$
\psi: X \backslash\{0\} \rightarrow \mathbf{G}(X, 1): x \mapsto \operatorname{span}\{x\}
$$

to define a hereditary $\sigma$ ideal $\mathscr{N}_{\mathbf{G}(X, 1)}$ on $\mathbf{G}(X, 1)$ in the following way:

$$
\mathscr{N}_{\mathbf{G}(X, 1)}=\mathscr{P}(\mathbf{G}(X, 1)) \cap\left\{E: \psi^{-1}(E) \in \mathscr{N}_{X}\right\} .
$$

Observe that $\psi^{-1}(E)$ is a cone: For every $v \in X, v \in \psi^{-1}(E)$ if and only if $r v \in \psi^{-1}(E)$ for every $r \in \mathbb{R} \backslash\{0\}$.

In case $X=\ell_{2}^{n}$ the Coarea Theorem $[\mathbf{9}, 3.4 .4$, Proposition 1] therefore implies that $E \in \mathscr{N}_{\mathbf{G}\left(\ell_{2}^{n}, 1\right)}$ if and only if $\mathscr{H}^{n-1}\left(\psi^{-1}(E) \cap S_{\ell_{2}^{n}}\right)=0$. Since the image of $\mathscr{H}^{n-1}$ under the canonical map $S_{\ell_{2}^{n}} \rightarrow \mathbf{G}\left(\ell_{2}^{n}, 1\right)$ is clearly an $\mathbf{O}(n)$ invariant, nontrivial, Borel finite measure, we conclude that $E \in \mathscr{N}_{\mathbf{G}\left(\ell_{2}^{n}, 1\right)}$ if and only if $\gamma_{n, 1}(E)=0$, see $[\mathbf{1 1}, 2.7 .16(6)]$.

\section{Proof of the Theorem}

2.1. Definition and properties of the coordinate functions. For each $j \in \mathbb{N}$ and each $k \in\left\{0,1, \ldots, 4^{j}-1\right\}$ we define $I_{j, k}=\mathbb{R} \cap\left\{t: k 4^{-j}<\right.$ $\left.t \leqslant(k+1) 4^{-j}\right\}$. Notice that for each $j,\left\{I_{j, k}: k=0,1, \ldots, 4^{j}-1\right\}$ is a partition of $I_{0,0}=(0,1]$. We next define a 1 periodic function $g: \mathbb{R} \rightarrow \mathbb{R}$ by its restriction to $I_{0,0}$ as follows:

$$
g(t)= \begin{cases}\frac{1}{2} & \text { if } t \in I_{1,0} \\ 0 & \text { if } t \in I_{1,1} \\ \frac{3}{4} & \text { if } t \in I_{1,2} \\ \frac{1}{4} & \text { if } t \in I_{1,3}\end{cases}
$$

Finally we let $f_{j}: I_{0,0} \rightarrow \mathbb{R}, j \in \mathbb{N} \backslash\{0\}$, be defined by the formula

$$
f_{j}(t)=\sum_{i=0}^{j-1} 4^{-i} g\left(4^{i} t\right)
$$

so that, in particular, $f_{1}=g \uparrow I_{0,0}$.

(A) For every $j \in \mathbb{N} \backslash\{0\}$ and every $k \in\left\{0,1, \ldots, 4^{j}-1\right\}$, the restriction of $f_{j}$ to $I_{j, k}$ is constant. In particular $f_{j}$ is Borel measurable. We let $c_{j, k} \in \mathbb{R}$ be defined by $\left\{c_{j, k}\right\}=f_{j}\left(I_{j, k}\right)$.

Proof: This is because $t \mapsto g\left(4^{i} t\right)$ itself is constant on each $I_{j, k}$ whenever $0 \leqslant i<j$. 
(B) For every $j \in \mathbb{N} \backslash\{0\}$ and every $k \in\left\{0,1, \ldots, 4^{j}-2\right\}$ one has

$$
\left|c_{j, k}-c_{j, k+1}\right| \geqslant \frac{1}{2 \cdot 4^{j-1}} .
$$

Proof: The initial case $j=1$ follows readily from the explicit definition of $f_{1}=g$ above. In fact, for the sake of this proof, it is useful to notice that if $s, t \in \mathbb{R}^{+} \backslash\{0\}$ belong to two distinct members of the partition $\{(m / 4,(m+1) / 4]: m \in \mathbb{N}\}$ and belong to the same interval $(k, k+1]$ for some $k \in \mathbb{N}$, then $|g(s)-g(t)| \geqslant \frac{1}{2}$. Also, $|g(s)-g(t)| \leqslant 1$ regardless of the relative position of $s$ and $t$.

Assuming the claim holds for $j$, we proceed to prove it for $j+1$. We notice that

$$
f_{j+1}(t)=f_{j}(t)+4^{-j} g\left(4^{j} t\right) .
$$

Letting $k \in\left\{0,1, \ldots, 4^{j}-2\right\}$, we distinguish between two cases. First suppose that $I_{j+1, k} \cup I_{j+1, k+1} \subseteq I_{j, k^{\prime}}$ for some $k^{\prime} \in\left\{0,1, \ldots, 4^{j}-1\right\}$. Letting $s \in I_{j+1, k}$ and $t \in I_{j+1, k+1}$ we infer from (3) that

$$
\begin{aligned}
\left|c_{j+1, k}-c_{j+1, k+1}\right| & =\left|c_{j, k^{\prime}}+4^{-j} g\left(4^{j} s\right)-c_{j, k^{\prime}}-4^{-j} g\left(4^{j} t\right)\right| \\
& =\frac{1}{4^{j}}\left|g\left(4^{j} s\right)-g\left(4^{j} t\right)\right| \\
& \geqslant \frac{1}{2 \cdot 4^{j}} .
\end{aligned}
$$

The second case occurs when $I_{j+1, k} \subseteq I_{j, k^{\prime}}$ and $I_{j+1, k+1} \subseteq I_{j, k^{\prime}+1}$ for some $k^{\prime} \in\left\{0,1, \ldots, 4^{j}-2\right\}$. Choosing $s \in I_{j+1, k}$ and $t \in I_{j+1, k+1}$ we infer from (3) and the induction hypothesis that

$$
\begin{aligned}
\left|c_{j+1, k}-c_{j+1, k+1}\right| & =\left|c_{j, k^{\prime}}+4^{-j} g\left(4^{j} s\right)-c_{j, k^{\prime}+1}-4^{-j} g\left(4^{j} t\right)\right| \\
& \geqslant\left|c_{j, k^{\prime}}-c_{j, k^{\prime}+1}\right|-4^{-j}\left|g\left(4^{j} s\right)-g\left(4^{j} t\right)\right| \\
& \geqslant \frac{1}{2 \cdot 4^{j-1}}-\frac{1}{4^{j}} \\
& \geqslant \frac{1}{2 \cdot 4^{j}} .
\end{aligned}
$$

(C) For every integers $1 \leqslant n<j$ one has $\sup \left\{\left|f_{j}(t)-f_{n}(t)\right|: t \in\right.$ $\left.I_{0,0}\right\} \leqslant 4^{-n}$. 
Proof: Given $t \in I_{0,0}$ and $i \in \mathbb{N} \backslash\{0\}$ it immediately follows from (3) that $\left|f_{i+1}(t)-f_{i}(t)\right| \leqslant \frac{3}{4} 4^{-i}$. Therefore,

$$
\left|f_{j}(t)-f_{n}(t)\right| \leqslant \sum_{i=n}^{j-1}\left|f_{i+1}(t)-f_{i}(t)\right| \leqslant \frac{3}{4} \sum_{i=n}^{\infty} 4^{-i}=4^{-n} .
$$

\subsection{A Borel measurable "curve" in $\ell_{\mathbf{2}}$. Here we assume that}

$$
\left\langle\beta_{j}\right\rangle_{j} \in \ell_{2}(\mathbb{N})
$$

and with it we associate

$$
\gamma: I_{0,0} \rightarrow \ell_{2}(\mathbb{N}): t \mapsto t e_{0}+\sum_{j=1}^{\infty} \beta_{j} f_{j}(t) e_{j},
$$

where $e_{0}, e_{1}, e_{2}, \ldots$ is the canonical orthonormal basis of $\ell_{2}(\mathbb{N})$. We also abbreviate $S=\operatorname{im} \gamma$.

In Subsection 2.3 we show $S$ has finite $\mathscr{H}^{1}$ measure. In Subsection 2.5 we consider further restrictions regarding the parameters $\left\langle\beta_{j}\right\rangle_{j}$ in order that $\gamma$ be approximately differentiable almost nowhere. This in turn implies $S$ is purely unrectifiable, Subsection 2.6. In Subsections 2.7 and 2.8 we show how to calibrate the parameters $\left\langle\beta_{j}\right\rangle_{j}$ so that all these conditions are met. Finally, in Subsection 2.10 we exhibit "many" lines in $\ell_{2}(\mathbb{N})$ on which $S$ projects to a nonnegligible set.

For now we observe that

(D) $\gamma$ is Borel measurable, and its image $S$ is Borel measurable as well.

Proof: Letting $P_{n}: \ell_{2}(\mathbb{N}) \rightarrow \ell_{2}(\mathbb{N})$ be the orthogonal projection onto $\operatorname{span}\left\{e_{0}, e_{1}, \ldots, e_{n}\right\}$ we notice that each $P_{n} \circ \gamma$ is Borel measurable, and that $\left\langle P_{n} \circ \gamma\right\rangle_{n}$ converges (uniformly) to $\gamma$. The Borel measurability of $\gamma$ easily follows, and in turn that of $S$ becomes a consequence of the injectivity of $\gamma$, see e.g. [22, Theorem 4.5.4].

\subsection{The Luzin (N) property of $\gamma$ and the Hausdorff measure of its image.}

For every subset $E \subseteq I_{0,0}$ one has $\mathscr{H}^{1}(\gamma(E)) \leqslant \mathscr{L}^{1}(E)$. In particular, $\mathscr{H}^{1}(\gamma(N))=0$ whenever $N \subseteq I_{0,0}$ and $\mathscr{L}^{1}(N)=0$. Furthermore $\mathscr{H}^{1}(S)=1$.

Proof: Letting as above $P_{0}$ denote the orthogonal projection on $\operatorname{span}\left\{e_{0}\right\}$ we notice that $P_{0}(S)=I_{0,0}$ and therefore $\mathscr{H}^{1}(S) \geqslant 1$. It thus remains only to show that $\mathscr{H}^{1}(S) \leqslant 1$. We shall first establish the inequality 
in case $E=I_{j_{0}, k_{0}}$ for some $j_{0} \in \mathbb{N}$ and $k_{0} \in\left\{0,1, \ldots, 4^{j_{0}}-1\right\}$. Given $n \geqslant j_{0}$ we define

$$
\tau_{n}=\sqrt{\sum_{j=n+1}^{\infty} \beta_{j}^{2}}
$$

We also let

$$
K_{n}=\left\{0,1, \ldots, 4^{n}-1\right\} \cap\left\{k: I_{n, k} \subseteq I_{j_{0}, k_{0}}\right\},
$$

so that readily $I_{j_{0}, k_{0}}=\cup_{k \in K_{n}} I_{n, k}$. With each $k \in K_{n}$ we associate the finite sequence of integers $k_{j} \in\left\{0,1, \ldots, 4^{j}-1\right\}, j=1, \ldots, n$, characterized by the relations $I_{n, k} \subseteq I_{j, k_{j}}$. Given a triple $n \in \mathbb{N} \backslash\{0\}, k \in K_{n}$, $t \in I_{n, k}$, we now define

$$
\begin{aligned}
x_{n, k}(t) & =t e_{0}+\sum_{j=1}^{n} \beta_{j} c_{j, k_{j}} e_{j}=t e_{0}+\sum_{j=1}^{n} \beta_{j} f_{j}(t) e_{j}, \\
y_{n, k} & =\sum_{j=n+1}^{\infty} \beta_{j} c_{n, k} e_{j}=\sum_{j=n+1}^{\infty} \beta_{j} f_{n}(t) e_{j},
\end{aligned}
$$

where the $c_{j, k}$ 's are defined in Subsection 2.1(A). Letting $P_{n}: \ell_{2}(\mathbb{N}) \rightarrow$ $\ell_{2}(\mathbb{N})$ still denote the orthogonal projection onto $\operatorname{span}\left\{e_{0}, e_{1}, \ldots, e_{n}\right\}$, and abbreviating $P_{n}^{\perp}=\operatorname{id}_{\ell_{2}}-P_{n}$, we next observe that

$$
P_{n}(\gamma(t))=x_{n, k}(t)
$$

and that

$$
\left\|P_{n}^{\perp}(\gamma(t))-y_{n, k}\right\|^{2}=\sum_{j=n+1}^{\infty} \beta_{j}^{2}\left|f_{j}(t)-f_{n}(t)\right|^{2} \leqslant\left(4^{-n}\right)^{2} \tau_{n}^{2},
$$

according to Subsection 2.1(C). In other words $\gamma\left(I_{n, k}\right) \subseteq S_{n, k}$ where

$$
\begin{aligned}
& S_{n, k}=\ell_{2}(\mathbb{N}) \cap\left\{z: P_{n}(z)=x_{n, k}(t) \text { for some } t \in I_{n, k}\right. \\
& \text { and } \left.\left\|P_{n}^{\perp}(z)-y_{n, k}\right\| \leqslant 4^{-n} \tau_{n}\right\} .
\end{aligned}
$$

Abbreviating $d_{n}=\operatorname{diam} S_{n, k}=4^{-n} \sqrt{1+4 \tau_{n}^{2}}$, it follows from the definition of Hausdorff measure that

$$
\begin{aligned}
\mathscr{H}_{d_{n}}^{1}\left(\gamma\left(I_{j_{0}, k_{0}}\right)\right) \leqslant & \sum_{k \in K_{n}} \operatorname{diam} S_{n, k} \\
& =\left(\operatorname{card} K_{n}\right) 4^{-n} \sqrt{1+4 \tau_{n}^{2}}=\mathscr{L}^{1}\left(I_{j_{0}, k_{0}}\right) \sqrt{1+4 \tau_{n}^{2}} .
\end{aligned}
$$

Letting $n \rightarrow \infty$ we conclude that

$$
\mathscr{H}^{1}\left(\gamma\left(I_{j_{0}, k_{0}}\right)\right) \leqslant \mathscr{L}^{1}\left(I_{j_{0}, k_{0}}\right) .
$$


Now if $E \subseteq I_{0,0}$ is arbitrary and $\varepsilon>0$ we choose an open set $U \subseteq \mathbb{R}$ containing $E$ and such that $\mathscr{L}^{1}(U)<\varepsilon+\mathscr{L}^{1}(E)$. We further extract a disjointed sequence $\left\langle J_{i}\right\rangle_{i}$ from the family $\left\{I_{j, k}: j \in \mathbb{N}\right.$ and $k=$ $\left.0,1, \ldots, 4^{j}-1\right\}$ such that $U \cap I_{0,0}=\cup_{i} J_{i}$. We note that

$$
\mathscr{H}^{1}(\gamma(E)) \leqslant \sum_{i} \mathscr{H}^{1}\left(\gamma\left(J_{i}\right)\right) \leqslant \sum_{i} \mathscr{L}^{1}\left(J_{i}\right)<\varepsilon+\mathscr{L}^{1}(E) .
$$

Since $\varepsilon$ is arbitrary the proof is complete.

2.4. The random variables $k_{j}$ and $\delta_{j}$. We define a countable set

$$
D=I_{0,0} \cap\left\{k 4^{-j}: j \in \mathbb{N} \text { and } k=1, \ldots, 4^{j}\right\},
$$

and for each $t \in I_{0,0} \backslash D$ we let $\left\langle k_{j}(t)\right\rangle_{j}$ be the unique sequence of integers such that $t \in I_{j, k_{j}(t)}$. We further define the relative distance of $t$ to the pair of endpoints of $I_{j, k_{j}(t)}$ by

$$
\delta_{j}(t)=\frac{\operatorname{dist}\left(t, \operatorname{bdry} I_{j, k_{j}(t)}\right)}{4^{-j}},
$$

and we notice that $0<\delta_{j}(t) \leqslant \frac{1}{2}$.

The $k_{j}$ and $\delta_{j}$ are clearly Borel measurable. Occasionally we will regard these as random variables on the probability space $\left(I_{0,0}, \mathscr{B}\left(I_{0,0}\right), \mathscr{L}^{1}\right)$, where $\mathscr{L}^{1}$ is the Lebesgue measure on the $\sigma$-algebra $\mathscr{B}\left(I_{0,0}\right)$ of Borel subsets of $I_{0,0}$. We observe that the random variables $k_{j} \bmod 4, j \in \mathbb{N}$, are mutually independent.

\subsection{Whether $\gamma$ is not approximately differentiable.}

If $t \in I_{0,0} \backslash D$ is so that

$$
\limsup _{j} \frac{\beta_{j}}{\delta_{j}(t)}=\infty
$$

then

$$
\operatorname{ap} \limsup _{s \rightarrow t} \frac{\|\gamma(s)-\gamma(t)\|}{|s-t|}=\infty .
$$

Proof: Given $\Lambda>0$ we define

$$
B_{\Lambda}=I_{0,0} \cap\left\{s: \frac{\|\gamma(s)-\gamma(t)\|}{|s-t|} \geqslant \Lambda\right\} .
$$

By definition of ap limsup we need to prove that $\Theta^{* 1}\left(\mathscr{L}^{1}\left\llcorner B_{\Lambda}, t\right)>\right.$ 0 . Hypothesis (H2) guarantees that there exists a subsequence $\left\langle\beta_{j(n)}\right\rangle_{n}$ of $\left\langle\beta_{j}\right\rangle_{j}$ such that

$$
\frac{\beta_{j(n)}}{\delta_{j(n)}(t)} \geqslant \Lambda
$$


for every $n$. We consider one $j=j(n)$ of these indices, which we assume sufficiently large for $0 \neq k_{j}(t) \neq 4^{j}-1$. For either $k=k_{j}(t)-1$ or $k=k_{j}(t)+1$ we have

$$
\mathscr{L}^{1}\left(B\left(t, 2 \delta_{j}(t) 4^{-j}\right) \cap I_{j, k}\right)=\delta_{j}(t) 4^{-j} .
$$

Since $I_{j, k_{j}(t)}$ and $I_{j, k}$ are consecutive it follows from Subsection 2.1(B) that

$$
\left|f_{j}(s)-f_{j}(t)\right| \geqslant \frac{1}{2 \cdot 4^{j-1}}
$$

whenever $s \in I_{j, k}$. If furthermore $|s-t| \leqslant 2 \delta_{j}(t) 4^{-j}$ then

$$
\frac{\left|f_{j}(s)-f_{j}(t)\right|}{|s-t|} \geqslant\left(\frac{1}{2 \cdot 4^{j-1}}\right)\left(\frac{1}{2 \delta_{j}(t) 4^{-j}}\right)=\frac{1}{\delta_{j}(t)} .
$$

Consequently, if $s \in B\left(t, 2 \delta_{j}(t) 4^{-j}\right) \cap I_{j, k}$ then

$$
\begin{array}{r}
\frac{\|\gamma(s)-\gamma(t)\|}{|s-t|}=\sqrt{1+\sum_{i=1}^{\infty} \frac{\beta_{i}^{2}\left|f_{i}(s)-f_{i}(t)\right|^{2}}{|s-t|^{2}}} \\
\geqslant \frac{\beta_{j}\left|f_{j}(s)-f_{j}(t)\right|}{|s-t|} \geqslant \frac{\beta_{j}}{\delta_{j}(t)} \geqslant \Lambda .
\end{array}
$$

Accordingly, $B\left(t, 2 \delta_{j}(t) 4^{-j}\right) \cap I_{j, k} \subseteq B_{\Lambda}$ and therefore

$$
\frac{\mathscr{L}^{1}\left(B_{\Lambda} \cap B\left(t, 2 \delta_{j}(t) 4^{-j}\right)\right)}{\mathscr{L}^{1}\left(B\left(t, 2 \delta_{j}(t) 4^{-j}\right)\right)} \geqslant \frac{\delta_{j}(t) 4^{-j}}{4 \delta_{j}(t) 4^{-j}}=\frac{1}{4} .
$$

Recalling this holds for each $j=j(n)$ and letting $n \rightarrow \infty$ we obtain

$$
\Theta^{* 1}\left(\mathscr{L}^{1}\left\llcorner B_{\Lambda}, t\right) \geqslant \frac{1}{4},\right.
$$

and the proof is complete.

\subsection{Whether $S$ is purely unrectifiable.}

If the parameters $\left\langle\beta_{j}\right\rangle_{j} \in \ell_{2}(\mathbb{N})$ verify

$$
\limsup _{j} \frac{\beta_{j}}{\delta_{j}(t)}=\infty \text { for } \mathscr{L}^{1} \text { almost every } t \in I_{0,0} \backslash D,
$$

then $S$ is purely $\left(\mathscr{H}^{1}, 1\right)$ unrectifiable.

Proof: Assume if possible that there exists a Lipschitz $\lambda: \mathbb{R} \supseteq A \rightarrow$ $\ell_{2}(\mathbb{N})$ such that $\mathscr{H}^{1}(S \cap \operatorname{im} \lambda)>0$. In view of Subsection 1.3 there is no 
restriction to assume that $A=[a, b]$ and that $\lambda$ is injective and an arclength parametrization. According to Subsection $1.2 \lambda$ is differentiable at each $s \in(a, b) \backslash N$ where $N \subseteq(a, b)$ is $\mathscr{L}^{1}$ negligible. We define

$$
S_{1}=(S \cap \operatorname{im} \lambda) \backslash \lambda(N \cup\{a, b\})
$$

and we notice $\mathscr{H}^{1}\left(S_{1}\right)=\mathscr{H}^{1}(S)>0$. For each $x \in S_{1}$ we let $s_{x} \in(a, b)$ be such that $x=\lambda\left(s_{x}\right)$ and we define a unit vector $v_{x}=\lambda^{\prime}\left(s_{x}\right)$. Routine verifications show that $v_{x}$ is tangent to $S_{1}$ in the following sense: For every $\varepsilon>0$ there exists $r(x, \varepsilon)>0$ such that

$$
\left\|\frac{y-x}{\|y-x\|}-\left( \pm v_{x}\right)\right\|<\varepsilon
$$

whenever $y \in S_{1} \cap B(x, r(x, \varepsilon)) \backslash\{x\}$.

We next define

$$
S_{2}=S_{1} \backslash(\gamma(D) \cup Z),
$$

where $D$ is defined in Subsection 2.4 and $Z \subseteq S_{1}$ consists of the points that are isolated in $S_{1} \backslash \gamma(D)$. We notice that $\mathscr{H}^{1}\left(S_{2}\right)=\mathscr{H}^{1}\left(S_{1}\right)>0$ because both $\gamma(D)$ and $Z$ are countable. We claim that for every $x \in S_{2}$ one has

$$
v_{x}= \pm e_{0}
$$

In order to prove this we choose a sequence $\left\langle y_{i}\right\rangle_{i}$ in $\left(S_{1} \backslash \gamma(D)\right) \backslash\{x\}$ converging to $x$ and we define $t, t+h_{i} \in I_{0,0} \backslash D$ uniquely by the relations $\gamma(t)=x$ and $\gamma\left(t+h_{i}\right)=y_{i}$. Given $j \geqslant 1$ we observe that

$$
\left\langle y_{i}-x, e_{j}\right\rangle=\left\langle\gamma\left(t+h_{i}\right)-\gamma(t), e_{j}\right\rangle=\beta_{j}\left(f_{j}\left(t+h_{i}\right)-f_{j}(t)\right) .
$$

As $\left|h_{i}\right| \leqslant\left\|y_{i}-x\right\| \rightarrow 0$ and $t \notin D$ we infer from Subsection 2.1(A) that $\left\langle y_{i}-x, e_{j}\right\rangle=0$ if $i$ is large enough. Therefore $\left\langle v_{x}, e_{j}\right\rangle=0$. Since $j \geqslant 1$ is arbitrary (5) is established.

Recall that $P_{0}: \ell_{2}(\mathbb{N}) \rightarrow \ell_{2}(\mathbb{N})$ denotes the orthogonal projection onto the line $\operatorname{span}\left\{e_{0}\right\}$, and $P_{0}^{\perp}=\mathrm{id}_{\ell_{2}}-P_{0}$. It follows from (4) and (5) that

$$
\left\|P_{0}^{\perp}(y-x)\right\|<\varepsilon\|y-x\|
$$

whenever $x \in S_{2}$ and $y \in S_{2} \cap B(x, r(x, \varepsilon))$. For the remaining part of this proof we fix $0<\varepsilon<1$. For each $k \in \mathbb{N} \backslash\{0\}$ we let

$$
S_{3, k}=S_{2} \cap\left\{x: r(x, \varepsilon)>\frac{1}{k}\right\} .
$$

Since $\mathscr{H}^{1}\left(S_{2}\right)>0$ and $S_{2}=\cup_{k=1}^{\infty} S_{3, k}$ there exists an integer $k$ such that $\mathscr{H}^{1}\left(S_{3, k}\right)>0$. We next infer from the Lindelöf property of $S_{3, k}$ that 
there exists $x_{0} \in S_{3, k}$ such that the set

$$
S_{4}=S_{3, k} \cap U\left(x_{0}, \frac{1}{2 k}\right)
$$

is not $\mathscr{H}^{1}$ negligible. Now if $x, y \in S_{4}$ then

(7) $\|y-x\| \leqslant\left\|P_{0}^{\perp}(y-x)\right\|+\left\|P_{0}(y-x)\right\|<\varepsilon\|y-x\|+\left\|P_{0}(y-x)\right\|$.

Multiplying (6) by $1-\varepsilon$ and plugging into (7) yields

$$
\left(\frac{1-\varepsilon}{\varepsilon}\right)\left\|P_{0}^{\perp}(y-x)\right\| \leqslant(1-\varepsilon)\|y-x\| \leqslant\left\|P_{0}(y-x)\right\| .
$$

Identifying $\operatorname{span}\left\{e_{0}\right\}$ with $\mathbb{R}$ in the obvious way, the above means that for every $s, t \in E=P_{0}\left(S_{4}\right)$ one has

$$
\|\gamma(s)-\gamma(t)\|^{2}=|s-t|^{2}+\left\|P_{0}^{\perp}(\gamma(s)-\gamma(t))\right\|^{2} \leqslant|s-t|^{2}\left(1+\left(\frac{\varepsilon}{1-\varepsilon}\right)^{2}\right) .
$$

We infer from Subsection 2.3 that $\mathscr{L}^{1}(E)>0$. Furthermore

$$
\operatorname{ap} \limsup _{s \rightarrow t} \frac{\|\gamma(s)-\gamma(t)\|}{|s-t|} \leqslant \sqrt{1+\left(\frac{\varepsilon}{1-\varepsilon}\right)^{2}}
$$

at each Lebesgue point $t$ of $E$. In view of our hypothesis (H3) and Subsection 2.5 , we have readily obtained the sought for contradiction.

2.7. We should now proceed to showing that there actually exists a choice of parameters $\left\langle\beta_{j}\right\rangle_{j}$ in $\ell_{2}(\mathbb{N})$ that verifies hypothesis (H3) of Subsection 2.6. This will be done in two steps. We start with the following observation.

\section{Assume that}

(1) $\left\langle\lambda_{n}\right\rangle_{n}$ is a sequence in $\mathbb{N} \backslash\{0\}$ and $\langle j(n)\rangle_{n}$ is the sequence defined by $j(1)=1$ and $j(n+1)=j(n)+\lambda_{n}=1+\sum_{k \leqslant n} \lambda_{k}$;

(2) $\sum_{n=1}^{\infty} 4^{-\lambda_{n}}=\infty$;

(3) $\left\langle\beta_{j}\right\rangle_{j} \in \ell_{2}(\mathbb{N})$ is so that

$$
\lim _{n} \beta_{j(n)} 4^{\lambda_{n}}=\infty
$$

It follows that

$$
\limsup _{j} \frac{\beta_{j}}{\delta_{j}(t)}=\infty
$$

for $\mathscr{L}^{1}$ almost every $t \in I_{0,0} \backslash D$. 
Proof: We define a partition $\mathbb{N} \backslash\{0\}=\cup_{n=1}^{\infty} J_{n}$ as follows:

$$
J_{n}=\{j(n), j(n)+1, \ldots, j(n+1)-1\} .
$$

Thus card $J_{n}=j(n+1)-j(n)=\lambda_{n}$. We also let

$$
\begin{aligned}
& A_{n}^{0}=\bigcap_{j \in J_{n}}\left\{t: k_{j}(t) \bmod 4=0\right\}, \\
& A_{n}^{3}=\bigcap_{j \in J_{n}}\left\{t: k_{j}(t) \bmod 4=3\right\},
\end{aligned}
$$

where $k_{j}$ are as in Subsection 2.4, and $A_{n}=A_{n}^{0} \cup A_{n}^{3}$. We notice that

$$
\mathscr{L}^{1}\left(A_{n}\right)=2 \cdot 4^{-\lambda_{n}} .
$$

Since the sequence $\left\langle A_{n}\right\rangle_{n}$ is independent, and since $\sum_{n=1}^{\infty} \mathscr{L}^{1}\left(A_{n}\right)=\infty$ according to hypothesis (2), it follows from the Borel-Cantelli Lemma [7, Theorem 8.3.4] that

$$
\mathscr{L}^{1}\left(\limsup _{n} A_{n}\right)=1
$$

Upon observing that

$$
A_{n}=\left\{\delta_{j(n)} \leqslant 4^{-\lambda_{n}}\right\},
$$

we infer that for $\mathscr{L}^{1}$ almost every $t \in I_{0,0} \backslash D$ the following holds: For every $m \in \mathbb{N}$ there exists $n \geqslant m$ such that $t \in A_{n}$, i.e. $\delta_{j(n)} \leqslant 4^{-\lambda_{n}}$. In particular,

$$
\frac{\beta_{j(n)}}{\delta_{j(n)}(t)} \geqslant \beta_{j(n)} 4^{\lambda_{n}}
$$

In view of hypothesis (3) the proof is complete.

\subsection{Calibrating the parameters $\left\langle\beta_{j}\right\rangle_{j}$.}

For every $\frac{1}{2}<\alpha<1$ the sequence $\left\langle\beta_{j}\right\rangle_{j} \in \ell_{2}(\mathbb{N})$ defined by $\beta_{j}=\left(\frac{1}{j}\right)^{\alpha}$ verifies hypothesis (H3) of Subsection 2.6.

Proof: We shall prove this by showing the hypotheses of Subsection 2.7 are satisfied for some appropriate choice of $\left\langle\lambda_{n}\right\rangle_{n}$. Notice $\left\langle\lambda_{n}\right\rangle_{n}$ should tend to $\infty$ in order for Subsection 2.7(3) to hold, but not too fast, according to Subsection 2.7(2). We define $\lambda_{n}=\left\lfloor\log _{4} n\right\rfloor$ for $n \geqslant 4$, and $\lambda_{1}=\lambda_{2}=\lambda_{3}=1$. Thus $4^{-\lambda_{n}} \geqslant \frac{1}{n}$ for $n \geqslant 4$, and Subsection 2.7(2) is readily verified. In order to show Subsection $2.7(3)$ holds as well, we need an upper bound for $j(n)$. Notice

$j(n) \leqslant j(n+1)=1+\sum_{k \leqslant n} \lambda_{k}=4+\sum_{k=4}^{n}\left\lfloor\log _{4} k\right\rfloor \leqslant 4+n \log _{4} n<C n \log n$ 
if $n$ is large enough, for some appropriate constant $C>0$. Upon noticing that $4^{\lambda_{n}} \geqslant \frac{n}{4}$ we infer that for large $n$

$$
\beta_{j(n)} 4^{\lambda_{n}} \geqslant\left(\frac{1}{C n \log n}\right)^{\alpha} \frac{n}{4}=\left(\frac{1}{4 C^{\alpha}}\right) \frac{n^{1-\alpha}}{(\log n)^{\alpha}}
$$

from which Subsection 2.7(3) follows at once.

2.9. For the remaining part of this paper we fix a choice of parameters $\left\langle\beta_{j}\right\rangle_{j}$ as in Subsection 2.8. This determines the map $\gamma: I_{0,0} \rightarrow \ell_{2}(\mathbb{N})$ and the set $S=\operatorname{im} \gamma$. We recall from Subsections 2.2, 2.3, and 2.6 that $S$ is Borel measurable, $\mathscr{H}^{1}(S)=1$, and $S$ is purely $\left(\mathscr{H}^{1}, 1\right)$ unrectifiable. We will next consider the collection of directions from which $S$ is visible, defined by

$$
G=\mathbf{G}\left(\ell_{2}, 1\right) \cap\left\{L: \mathscr{H}^{1}\left(P_{L}(S)\right)>0\right\} .
$$

\subsection{0. $S$ is visible from Aronszajn nonnegligeably many direc- tions.}

Assume that

(A) $\langle\eta\rangle_{j} \in \ell_{1}(\mathbb{N}), 0<\eta_{j}<\frac{1}{2}$ for every $j \in \mathbb{N}$, and $\sum_{j \in \mathbb{N}} \eta_{j}<\frac{1}{2}$;

(B) $0<\varepsilon<1$;

(C) $\langle\theta\rangle_{j} \in \ell_{2}(\mathbb{N})$ and $\|\theta\|_{\ell_{2}} \leqslant \frac{1-\varepsilon}{2\|\beta\|_{\ell_{2}}}$;

and define

$$
G_{\eta, \theta, \varepsilon}=S_{\ell_{2}} \cap\left\{u:\left|u_{0}-1\right| \leqslant \varepsilon \text { and }\left|u_{j}\right| \leqslant \frac{\eta_{j} \theta_{j}}{4^{j}} \text { for all } j \geqslant 1\right\} .
$$

It follows that

(D) $\psi\left(G_{\eta, \theta, \varepsilon}\right) \subseteq G$ (where $G$ is defined in Subsection 2.9 and $\psi$ is defined in Subsection 1.4);

(E) $\psi\left(G_{\eta, \theta, \varepsilon}\right) \notin \mathscr{N}_{\mathbf{G}\left(\ell_{2}, 1\right)}$ (where $\psi$ and $\mathscr{N}_{\mathbf{G}\left(\ell_{2}, 1\right)}$ are defined in Subsection 1.4).

Proof: Given $u \in S_{\ell_{2}}$ we let $h_{u}: I_{0,0} \rightarrow \mathbb{R}$ be defined by $h_{u}(t)=\langle\gamma(t), u\rangle$ and we observe that $u \in G$ if and only if $\mathscr{L}^{1}\left(h_{u}\left(I_{0,0}\right)\right)>0$. In order to establish this for those $u \in G_{\eta, \theta, \varepsilon}$ we decompose $h_{u}(t)=u_{0} t+\rho_{u}(t)$ where

$$
\rho_{u}(t)=\sum_{j=1}^{\infty} \beta_{j} u_{j} f_{j}(t) .
$$

The point will be to infer from the smallness of the $u_{j}$ 's that $\rho_{u}$ is Lipschitz (with small Lipschitz constant) when restricted to a suitable 
nonnegligible subset $A$ of $I_{0,0}$. We let $A_{j}=\left\{t: \delta_{j}(t) \geqslant \eta_{j}\right\}$ for each $j \in$ $\mathbb{N}$, and

$$
A=\bigcap_{j \in \mathbb{N}} A_{j}
$$

Observe that $\mathscr{L}^{1}\left(I_{0,0} \backslash A_{j}\right)=2 \eta_{j}$, thus

$$
1-\mathscr{L}^{1}(A)=\mathscr{L}^{1}\left(I_{0,0} \backslash \bigcap_{j \in \mathbb{N}} A_{j}\right)=\mathscr{L}^{1}\left(\bigcup_{j \in \mathbb{N}} I_{0,0} \backslash A_{j}\right) \leqslant 2 \sum_{j \in \mathbb{N}} \eta_{j} .
$$

It therefore ensues from hypothesis $(\mathrm{A})$ that $\mathscr{L}^{1}(A)>0$.

We now turn to estimating $\operatorname{Lip} \rho_{u}\left\lceil A\right.$. Fix $j \geqslant 1$ and assume $s, t \in A_{j}$. If $s, t \in I_{j, k}$ for some $k \in\left\{0,1, \ldots, 4^{j}-1\right\}$ then $f_{j}(s)=f_{j}(t)$ according to Subsection 2.1(A). If not, the definition of $A_{j}$ implies $|s-t| \geqslant 4^{-j}\left(\delta_{j}(s)+\right.$ $\left.\delta_{j}(t)\right) \geqslant 2 \eta_{j} 4^{-j}$. In both cases

$$
\frac{\left|f_{j}(s)-f_{j}(t)\right|}{|s-t|} \leqslant \frac{4^{j}}{\eta_{j}} .
$$

Therefore $\operatorname{Lip} f_{j} \uparrow A \leqslant \operatorname{Lip} f_{j} \uparrow A_{j} \leqslant \frac{4^{j}}{\eta_{j}}$. In turn,

$$
\operatorname{Lip} \rho_{u}\left\lceil A \leqslant \sum_{j=1}^{\infty} \beta_{j}\left|u_{j}\right|\left(\frac{4^{j}}{\eta_{j}}\right) \leqslant \sum_{j=1}^{\infty} \beta_{j}\left|\theta_{j}\right| \leqslant\|\beta\|_{\ell_{2}}\|\theta\|_{\ell_{2}} \leqslant \frac{1-\varepsilon}{2} .\right.
$$

It next follows from the triangle inequality that

$$
\left(\frac{1-\varepsilon}{2}\right)|s-t| \leqslant\left|h_{u}(s)-h_{u}(t)\right| \leqslant 2|s-t|
$$

whenever $s, t \in A$ and $u \in G_{\eta, \theta, \varepsilon}$. In other words $A$ and $h_{u}(A)$ are lipeomorphic. Thus $\mathscr{L}^{1}\left(h_{u}(A)\right)>0$ and the proof of conclusion (D) is complete.

In order to establish conclusion (E), we start by showing that $\psi^{-1}\left(\psi\left(G_{\eta, \theta, \varepsilon}\right)\right)$ contains a cube $K_{\eta, \theta, \hat{\varepsilon}}$ defined by

$$
K_{\eta, \theta, \hat{\varepsilon}}=\mathbb{R}^{\mathbb{N}} \cap\left\{v:\left|v_{0}-1\right| \leqslant \hat{\varepsilon} \text { and }\left|v_{j}\right| \leqslant \hat{\varepsilon}\left(\frac{\eta_{j} \theta_{j}}{4^{j}}\right) \text { for all } j \geqslant 1\right\} \subseteq \ell_{2}(\mathbb{N}),
$$

provided $\hat{\varepsilon}>0$ is chosen small enough. Given $v \in K_{\eta, \theta, \hat{\varepsilon}}$ we let $r=$ $\|v\|$ and we note that $|1-\|v\|| \leqslant C(\|\beta\|) \hat{\varepsilon}$. Thus $u=r^{-1} v$ readily belongs to $G_{\eta, \theta, \varepsilon}$ insofar as $\hat{\varepsilon}$ is appropriately small. It then remains to classically remark that $K_{\eta, \theta, \hat{\varepsilon}}$ is not Aronszajn null. This is because it is homeomorphic to the Polish space

$$
C=[1-\hat{\varepsilon}, 1+\hat{\varepsilon}] \times\left(\prod_{j=1}^{\infty}\left[-\frac{\hat{\varepsilon} \eta_{j} \theta_{j}}{4^{j}}, \frac{\hat{\varepsilon} \eta_{j} \theta_{j}}{4^{j}}\right]\right)
$$


and therefore carries a Borel probability measure $\mu=\otimes_{j=0}^{\infty} \mu_{j}$ where each $\mu_{j}$ is a normalized Lebesgue measure supported on the $j^{\text {th }}$ compact interval factor. If $K_{\eta, \theta, \hat{\varepsilon}}$ were Aronszajn null, it would decompose into a countable union of Borel sets $B_{j}, j \in \mathbb{N}$, such that $\mathscr{H}^{1}\left(B_{j} \cap(y+\right.$ $\left.\left.\operatorname{span}\left\{e_{j}\right\}\right)\right)=0$ for every $y \in \operatorname{span}\left\{e_{j}\right\}^{\perp}$, and therefore also $\mu_{j}\left(B_{j} \cap(y+\right.$ $\left.\left.\operatorname{span}\left\{e_{j}\right\}\right)\right)=0$. Fubini's Theorem and the Borel measurability of $B_{j}$ then imply $\mu\left(B_{j}\right)=0$. Since $j$ is arbitrary we conclude $\mu\left(K_{\eta, \theta, \hat{\varepsilon}}\right)=0$, a contradiction.

\section{References}

[1] F. Almgren, Deformations and multiple-valued functions, in: "Geometric Measure Theory and the Calculus of Variations" (Arcata, Calif., 1984), Proc. Sympos. Pure Math. 44, Amer. Math. Soc., Providence, RI, 1986, pp. 29-130. DOI: 10.1090/pspum/044/ 840268.

[2] Y. Benyamini and J. Lindenstrauss, "Geometric Nonlinear Functional Analysis", Vol. 1, American Mathematical Society Colloquium Publications 48, American Mathematical Society, Providence, RI, 2000.

[3] A. S. Besicovitch, On the fundamental geometrical properties of linearly measurable plane sets of points (III), Math. Ann. 116(1) (1939), 349-357. DOI: 10.1007/BF01597361.

[4] P. Bouafia And T. De Pauw, Integral geometric measure in separable Banach space, Math. Ann. 363(1-2) (2015), 269-304. DOI : $10.1007 / \mathrm{s} 00208-014-1165-9$.

[5] M. CsöRnyeI, Aronszajn null and Gaussian null sets coincide, Israel J. Math. 111(1) (1999), 191-201. DOI: 10.1007/BF02810684.

[6] G. David And S. Semmes, Uniform rectifiability and quasiminimizing sets of arbitrary codimension, Mem. Amer. Math. Soc. 144, no. $687,(2000), 132$ pp. DOI: $10.1090 / \mathrm{memo} / 0687$.

[7] R. M. Dudley, "Real Analysis and Probability", The Wadsworth $\&$ Brooks/Cole Mathematics Series, Wadsworth \& Brooks/Cole Advanced Books \& Software, Pacific Grove, CA, 1989.

[8] R. Engelking, "General Topology", Translated from the Polish by the author, Monografie Matematyczne 60, [Mathematical Monographs 60], PWN-Polish Scientific Publishers, Warsaw, 1977.

[9] L. C. Evans And R. F. Gariepy, "Measure Theory and Fine Properties of Functions", Studies in Advanced Mathematics, CRC Press, Boca Raton, FL, 1992. 
[10] H. Federer, Dimension and measure, Trans. Amer. Math. Soc. 62(3) (1947), 536-547. DOI: 10.1090/S0002-9947-1947-0023325-3.

[11] H. FeDERER, "Geometric Measure Theory", Die Grundlehren der mathematischen Wissenschaften 153, Springer-Verlag New York Inc., New York, 1969.

[12] H. FEDERER, Flat chains with positive densities, Indiana Univ. Math. J. 35(2) (1986), 413-424. DOI: 10.1512/iumj.1986.35.35025.

[13] H. Federer And W. H. Fleming, Normal and integral currents, Ann. of Math. (2) 72(3) (1960), 458-520. DOI: $10.2307 / 1970227$.

[14] W. H. Fleming, Flat chains over a finite coefficient group, Trans. Amer. Math. Soc. 121 (1966), 160-186. DOI: 10.1090/S0002-99471966-0185084-5.

[15] W. B. Johnson, J. Lindenstrauss, and G. Schechtman, Extensions of Lipschitz maps into Banach spaces, Israel J. Math. 54(2) (1986), 129-138. DOI: $10.1007 /$ BF02764938.

[16] A. B. Kharazishvili, "Nonmeasurable Sets and Functions", North-Holland Mathematics Studies 195, Elsevier Science B.V., Amsterdam, 2004.

[17] B. KirChHeim, Rectifiable metric spaces: local structure and regularity of the Hausdorff measure, Proc. Amer. Math. Soc. 121(1) (1994), 113-123. DOI : $10.2307 / 2160371$.

[18] P. Mattila, "Geometry of Sets and Measures in Euclidean Spaces. Fractals and Rectifiability", Cambridge Studies in Advanced Mathematics 44, Cambridge University Press, Cambridge, 1995. DOI: $10.1017 /$ CB09780511623813.

[19] E. J. Mickle, On a decompostion theorem of Federer, Trans. Amer. Math. Soc. 92(2) (1959), 322-335. DOI: 10.1090/S00029947-1959-0112947-5.

[20] T. C. O'NeiL, A local version of the projection theorem, Proc. London Math. Soc. (3) 73(1) (1996), 68-104. DOI: 10.1112/plms/ s3-73.1.68.

[21] B. Solomon, A new proof of the closure theorem for integral currents, Indiana Univ. Math. J. 33(3) (1984), 393-418. DOI: 10.1512/iumj.1984.33.33022.

[22] S. M. SRIVAstava, "A Course on Borel Sets", Graduate Texts in Mathematics 180, Springer-Verlag, New York, 1998. DOI : 10.1007/ 978-3-642-85473-62.

[23] T. TAO, A quantitative version of the Besicovitch projection theorem via multiscale analysis, Proc. Lond. Math. Soc. (3) 98(3) (2009), 559-584. DOI: 10.1112/plms/pdn037. 
[24] B. White, A new proof of the compactness theorem for integral currents, Comment. Math. Helv. 64(2) (1989), 207-220. DOI: 10.1007/BF02564671.

[25] B. White, A new proof of Federer's structure theorem for $k$-dimensional subsets of $\mathbf{R}^{N}$, J. Amer. Math. Soc. 11(3) (1998), 693-701. DOI : $10.1090 /$ S0894-0347-98-00267-7.

Institut de Mathématiques de Jussieu

Paris Rive Gauche

UMR 7586, Equipe Géométrie et Dynamique

Bâtiment Sophie Germain, Case 7012

75205 Paris Cedex 13

France

E-mail address: thierry.de-pauw@imj-prg.fr

Rebut el 20 de maig de 2015. 